\title{
Facial Automaton for Conveying Emotions as a Social Rehabilitation Tool for People with Autism
}

\author{
Giovanni Pioggia, Maria Luisa Sica, Marcello Ferro, Silvia Casalini, \\ Roberta Igliozzi ${ }^{1}$, Filippo Muratori ${ }^{1}$, Arti Ahluwalia, Danilo De Rossi \\ Interdepartmental Research Center "E. Piaggio", University of Pisa, Italy \\ ${ }^{1}$ Scientific Institute Stella Maris (IRCCS), Pisa, Italy
}

\section{Introduction}

The terms Autistic Spectrum Disorders (ASD) and pervasive developmental disorders refer to a wide continuum of associated cognitive and neuro-behavioral disorders, including, but not limited to, three core-defining features: impairments in socialization, impairments in verbal and nonverbal communication, and restricted and repetitive patterns of behaviors. Although autism was first described over 50 years ago, our improved understanding of this complex disorder has emerged over the past two decades. Despite recent intense focus on autism, its study continues to be an art and science in fast evolution. There is marked variability in the severity of symptomatology across patients, and level of intellectual function can range from profound mental retardation through the superior range on conventional IQ tests. In ASD we can find: marked impairment in the use of multiple nonverbal behaviors, such as eye-to-eye gaze, facial expression, body posture, and gestures to regulate social interaction. The communication impairments seen in the autistic spectrum are far more complex than

presumed by simple speech delay and share some similarities with the deficits seen in children with developmental language disorders or specific language impairments. Young autistic $\stackrel{\oplus}{\subseteq}$ children, even if verbal, almost universally have comprehension deficits, in particular deficits 드 in understanding higher order complex questions. Deficits in pragmatics, the use of language O to communicate effectively, are almost universally present too. Another core characteristic of ASD is the presence of stereotyped behaviors and circumscribed/unusual interests, which encompass qualitative deficits in several aspects of behavior. It is moreover well documented that individuals with autism have impairments in processing of social and emotional \$ information, as evident in tasks assessing face and emotion recognition, imitation of body (O) movements, interpretation and use of gestures and theory of mind [Baron-Cohen 1994, Davies 1994, Dawson 1998, Smith 1994]. Typically developing infants show preferential attention to ocial rather than inanimate stimuli; in contrast, individuals with autism seem to lack these arly social predispositions [Spelke 1995, Maestro 2002]. This hypothesis was recently substantiated in a neurofunctional study [Schultz 2000] of face perception in autism, in which adequate task performance was accompanied by abnormal ventral temporal cortical activities, which in turn suggested that participants had treated faces as objects. Klin et al. [Klin 2002] $\bar{\Phi}$ created an experimental paradigm to measure social functioning in natural situations, in which they used eye-tracking technology to measure visual fixations of cognitively able 
individuals with autism. When viewing naturalistic social situations, people with autism demonstrate abnormal patterns of social visual pursuit consistent with reduced salience of eyes and increased salience of mouth, bodies and objects. In addition, individuals with autism use atypical strategies when performing such tasks, relying on individual pieces of the face rather than on the overall configuration. Alongside these perceptual anomalies, individuals with autism have deficits in conceiving other people's mental states or "mindblindness" [Baron-Cohen 2000]. The cognitive theory of mindblindness [Baron-Cohen 1997] suggests that individuals with autism have difficulty in conceiving of people as mental agents and cannot adequately perceive another persons mental state. This often leads to typically inappropriate reactions or behaviours in a variety of social encounters. Recent studies have shown that individuals, particularly those with high functioning autism, can learn to cope with common social situations if they are made to enact possible scenarios they may encounter. By recalling appropriate modes of behavior and expressions in specific situations, they are able to react appropriately. There are now a number of highly structured therapeutic approaches based on emotion recognition and social skill training using photographs, drawings, videos or DVDROMs (for example Mind Reading, produced by Human Emotions, UK). Currently, these treatment approaches suggested the use of robotic systems in order to encourage children with autism to take initiative and to interact with the robotic tools.

The use of the robotic technology aimed to help autistic subjects in everyday life began in 1976 with the work of Sylvia Weir and Ricky Emanuel [Weir 1976]. They used a mobile turtle-like robot, LOGO, able to interact with a patient within a highly structured environment. More recently François Michaud [Michaud 2003, Michaud 2002] and his research team at the University of Sherbrooke, investigated the use of mobile robots as a treatment tool. They tested several robots, different in shape, color and behavior, in order to study the main characteristics that may capture the attention of people with autism. They obtained important insights for the comprehension of human-robot interaction in autism sustaining the robot hypothesis as useful. People with autism focus their attention on single details, but the interaction with a robot may allow an autistic subject to concentrate herself/himself on the limited number of communication modalities of the robot. In addition, while the stress of the learning with a teacher can be detrimental, the interaction with a robot, which often the young patients associate with media and/or cinema characters, can reduce the emotional pressure allowing the child to better learn from the environment.

A more structured approach to the use of autonomous robots is AURORA (AUtonomous RObotic platform as a Remedial tool for children with Autism) [Dautenhahn 2002a, Dautenhahn 2002b]. AURORA represents the first systematic study on a therapeutic approach of robots in autism. People with autism are invited to interact in coordinated and synchronized social actions with the robots and the environment. In AURORA, behaviorbased architectures for the use of different robotic platforms such as mobile and humanoid robots (Robota developed by Aude Billard [Billard 2002]) were developed.

Recently the development of emotional cognitive architectures allowed the interaction to be based on empathy, e.g. the KISMET [Breazeal 2001] project developed by Cynthia Breazeal at the Robotic Life Group of MIT Media Lab, INFANOID or KEEPON [Kozima 2004, Kozima 2002] developed by Hideki Kozima at the National Institute of Information and Communications Technology (NICT) of Japan. INFANOID is an upper-torso child-like robot, capable of pointing, grasping, and of expressing a variety of gestures, while KEEPON is a simple yellow snowman-like robot, both equipped by eye-contact and joint attention functions. 
FACE (Facial Automaton for Conveying Emotions) [Pioggia 2004, Pioggia 2005] on the other hand follows a biomimetic approach. In FACE the biological behaviour is mimicked by means of dedicated smart soft materials and structures, intelligent control strategy, algorithms and artificial neural networks. It is part of an innovative android-based treatment which focuses on core aspects of the autistic disorder, namely social attention and the recognition of emotional expressions. FACE is a social believable artifact able to interact with the external environment, interpreting and conveying emotions through non verbal communication. FACE captures expressive and psychophysical correlates from its interlocutor and actuates behaviours with kinesics, a non verbal communication conveyed by body part movements and facial expressions. In the framework of A social therapy, FACE can act as an interface between a patient and a trained therapist in a specially equipped room. Both physiological and behavioural information is acquired in real time by means of an unobtrusive sensorized wearable interface from the patient during the treatment. This approach provides a structured environment that people with autism could consider to be "social", helping them to accept the human interlocutor and to learn through imitation. On the basis of a dedicated therapeutic protocol, FACE is able to engage in social interaction by modifying its behaviour in response to the patient's behaviour. Following an imitation-based learning strategy, we hope to verify that such a system can help children with autism to learn, interpret and use emotional information. If such learned skills can be extended to a social context, the whole FACE system will serve as an invaluable therapeutic tool for ASD, which we call FACE-T (T as in "therapy"). The FACE-T system consists of FACE itself, a sensorised life-shirt and the therapeutic protocol.

\section{Social interaction and communication}

Since the first days of our lives we are social beings. Children meet people's gaze, turn towards a voice, catch mom or dad's fingers and smile, but children affected by ASD show difficulties in taking part in such daily social interaction and communication with others. Children affected by ASD have impairment in the use of multiple nonverbal behaviors, such as eye-to-eye gaze, facial expression, body posture, and gestures. Young autistic children, even if verbally skilled, almost universally have comprehension and language communication deficits, i.e. they lack skilful use of language to communicate effectively. The presence of stereotyped behaviors and circumscribed interests can be also underlined. Meltzoff argued that an underdevelopment of the social communication can be explained in terms of imitation impairment [Meltzoff 1995]. Children usually imitate the behaviour of an interlocutor, children with autism do not; causing serious consequences. Early imitation is one of the most important instruments for the social learning, as well as innate to humans [Rizzolatti 1999]. An essential prerequisite for imitation is a connection between the sensory systems and the motor systems such that percepts can be mapped onto appropriate actions. This mapping is a difficult computational process as visual perception takes place in a different coordinate frame from motor control. This process is also more complex than pure object recognition since it requires integration of multiple objects (i.e., several limbs), their spatial relations, their relative and absolute movements, and even the intention of these movements.

The possible connection to imitation, however, came with the discovery of the mirror neurons [Rizzolatti 1999], a new class of visuomotor neurons recently discovered in the monkey's premotor cortex (F5 area). These neurons respond both when a particular action is performed by the recorded monkey and when the same action, performed by another 
individual, is observed [Pellegrino 1992]. From imaging and transcranial magnetic stimulation studies, there is also a great deal of evidence that a similar mirror system exists in humans [Decety 1994, Fadiga 1995, Decety 1996]. Surprisingly, this system seems to involve the Brocas area [Rizzolatti 1998], a brain region normally associated with speech production. The possible homology of F5 in monkey and Brocas area in humans led some authors [Rizzolatti 1998] to speculate that the ability to imitate actions and to understand them could have subserved the development of communication skills. This idea is consistent with Meltzoff and Moores [Meltzoff 1995] works and interpretations. Gallese and Goldman [Gallese 1998] suggest rather that mirror neurons participate in mind reading, a process accomplished by using ones own mental apparatus to predict the psychological state of others through mental simulations.

Rizzolatti pointed out that the process of imitation plays a crucial role in distinguishing between actions arising from within or actions induced by others [Rizzolatti 1999]. Imitation paves the way to the comprehension of the intentions of others establishing a reciprocal non verbal communication process in which the roles of imitator and model are continuously exchanged [Nadel 2004a, Nadel 2004b]. Moreover, in the early years, imitation plays a fundamental role for the emergence of proprioception, of the perception of the external world and of the ability to act our own actions. Enhancing the imitation skills of children through specifically designed treatments based on imitation may yield to an improvement in social development. Recently it has been proposed that the characteristic impairments of ASD, including deficits in imitation, theory of mind and social communication, can be caused by a dysfunction of the mirror neuron system [Dapretto 2005].

However, what strategy can be used to control and enhance the emergence of humanandroid imitation? As a first step, our idea was the realization of a structure capable of creating its own representations of the surrounding environment. It is an associative memory through which it may be possible to navigate within a behavioural space. These characteristics are typical of some areas of the central nervous system. This led us to abandon the idea of realising a controller based solely on a group of neurons in various states of connection. Furthermore, preformism impedes the topological and geometrical structure from developing in an adaptive manner. As a first approach, we used the model developed by Izhikevich [Izhikevich 2003] and a learning model based on the Theory of Neuronal Group Selection (TNGS) of G. Edelman [Edelman 1993]. As a first approach the learning process in FACE is based on imitating predefined stereotypical behaviours which can be represented in terms of FAPs (Fixed Action Patterns) followed by a continuous interaction with its environment. FAPs can be classified as belonging to action schemes, partly fixed on the basis of physical constraints and sensory-motor reflexes, partly subjected to a specialization on the basis of the experience. FACE is therefore able to continually learn, to adapt and evolve within a simplified behavioural space as a function of the environment and to maintain spontaneous activity open to any innovative and intelligible behaviours arising which may then be interpreted.

\section{Non verbal communication and autism}

Non verbal communication is usually understood as the process of sending and receiving wordless messages. Such messages can be communicated through gesture; body language or posture; facial expression and eye contact; prosodic features of speech such as intonation and stress and other paralinguistic features of speech such as voice quality, emotion and speaking 
style. Non verbal communication can occur through any sensory channel sight, sound, smell, touch or taste. Also, non-verbal communication comes in many forms at the same time. For example, a person's dress, tone of voice, attitude, and movement all contribute to the communication going on in a certain situation. The communication problems of autism vary depending on the intellectual and social development of the individual. Some may be unable to speak, whereas others may have rich vocabularies and are able to talk about topics of interest in great depth. Despite this variation, the majority of autistic individuals have little or no problem with pronunciation. Most have difficulty effectively using language. Many also have problems with word and sentence meaning, intonation, and rhythm. Those who can speak often say things that have no content or information. For example, an autistic individual may repeatedly count from one to five. Most autistic individuals do not make eye contact and have poor attention duration. They are often unable to use gestures either as a primary means of communication, as in sign language, or to assist verbal communication, such as pointing to an object they want. For many, speech and language develop, to some degree, but not to a normal ability level. This development is usually uneven.

\section{Instruments of diagnostic observation}

At the present time no "objective" procedure exists for the diagnosis of autism. Clinicians must, accordingly, rely on their clinical judgement, aided by guides to diagnosis such as DSM-IV (APA, 1994) and ICD-10 (World Health Organization, 1993), as well as by the results of various assessment instruments such as checklists, interviews, rating scales and observation schedules. Thus, an important help for all mental health operators that work with this disorder is given by standardised instruments that can facilitate in "communicating" to one another from a similar "point of view" during the diagnostic process. Besides this, these are very useful instruments that can create comparability across clinicians and researchers, as well as instruments that can individuate more homogeneous groups for research: Autism Diagnostic Interview - Revised (ADI-R) [Lord, Rutter \& Le Couter, 1994] and Autism Diagnostic Observation Schedule Generic (ADOS-G) [Lord, Risi, Lambrecht, Cook, Leventhal et al. 2000] are complementary instruments currently defined as the "gold standard" diagnostic instruments.

ADI-R is a standardised, semi structured, investigator-based interview for caregivers of autistic individuals, which provides a diagnostic algorithm for the ICD-10 and DSM-IV definition of autism. The focus is upon obtaining detailed descriptions of real behaviours and then establishing typicality and pervasiveness for diagnosis of autism. We find three sections about specific areas related to the diagnosis of autism: Communication and language (with different questions about verbal and non-verbal aspects of communication); Social development and play (in which interviewer asks about the presence, for example, of shared behaviours, emotional reciprocity, etc); and repetitive and stereotyped behaviours and unusual interests.

ADOS-G is an instrument that collects standard information about a child's behaviour in response to a predetermined schedule of activities. Activities and behaviours are taken from empirical research in autism and child development and refer to social and communicative features.

The CARS scale (Childhood Autism Rating Scales) [Schopler, et al., 1988] was developed in order to aid in the diagnostic process but it is also used to assess changes in autistic symptomatology at two/three years, at one year and at 6 month intervals. More recently it has been used also in shorter longitudinal studies. The CARS scale is subdivided in 15 items, relative to the main behavioural areas; it is assigned a variable score from 1 to 4 to every item; the score 1 indicates a behavior appropriate to the age, while a score 4 indicates 
an abnormal behavior. The total score obtained after the CARS test has an undeniable diagnostic and clinical usefulness. Inquiring the score of the single items of the test it is however possible to characterize other patient behaviors.

\section{FACE, the biomimetic social interactive machine}

The realisation of a social interactive machine entails critical requirements for its body, its sensory perception system, its mobility and its ability to perform tasks. The human mind responds and modifies itself with respect to the real world enabling the body to perceive, to act and to survive; human intelligence arises primarily from the interpretation of the body's needs. For this reason we preferred to follow a mind-body monism, i.e. an embodied mind able to perform the processing phase taking into account the domain of experiences where the machine is placed; such processes influence and are influenced by its own presence.

Dynamic interaction mechanisms are needed in order to place the android inside its environment: FACE is provided with extrinsic perception in order to interiorize the external world and to be able to suitably react. It possesses body structures as a support to the intrinsic perception (proprioception) and motor activity. The formation of a relationship domain close to a human context underlies the need of a high degree of believability in the FACE robot. Furthermore the robot has a space-time capability for both egocentricity and allocentricity, taking into account the actuation of preprogrammed behaviours as well as an imitative learning strategy.

The android FACE consists of a passive articulated body equipped with a believable facial display system based on biomimetic engineering principles. The latest prototype of the FACE robot is shown in Figure 1. Its head consists of an artificial skull covered by an artificial skin which is a thin silicone-based mask equipped with sensory and actuating system. The mask is fabricated by means of life-casting techniques and aesthetically represents a copy of the head of a subject, both in shape and texture. An artificial muscular architecture and servomotors allow FACE to express and modulate the six basic emotions (happiness, sadness, surprise, anger, disgust, fear) in a repeatable and flexible way. This process can be controlled thanks to an artificial skin consisting of a 3D latex foam equipped with a biomimetic system of proprioceptive mapping described in section 6.1. This structure allows the expression required to be achieved by means of a trial and error process. The artificial skin covers an artificial skull which is equipped with an actuating system.

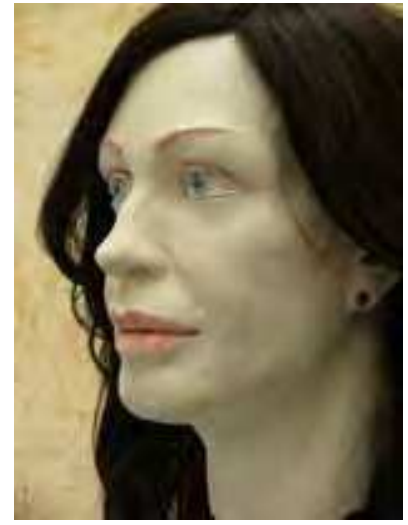

Fig. 1. The latest prototype of FACE. 
FACE can enable real-time acquisition of both physiological and behavioural information by means of an unobtrusive sensorized wearable interface from the interlocutor. It is able to analyse the emotional reactions of individuals through optical analyses of facial expressions, to track a human face over time and to automatically store all data (described in section 6.2). The robot's eyes are realised using animatronic techniques and their expressiveness is achieved through an artificial muscular structure surrounding the orbital region. It sees differently from man, using stereoscopic vision over frequency rather than over space. A three-dimensional contouring apparatus, equipped with a section for data analysis, rebuilds an internal representation of a portion of the world before it. Currently FACE surveys the curvature of the three dimensional scene once per second. We adopted a neural approach to allow FACE to recognize the expression of a subject. A dedicated process detects a number of points (markers), which are used to divide the human face into four main areas (left eye, right eye, nose and mouth). The data of each area are processed by a hierarchical neuralnetwork architecture based on Kohonen self organizing maps and a multi-layer perceptron.

\section{Perception and learning of the human behavior}

In human-machine social interaction, the intersection between biology and engineering needs a context which enables the conditions for the development of adapting dynamics. Perception and learning of the dynamics of human-machine interactions play an important role in social verbal and nonverbal communication. In order to enable continuous learning and adaptation within a simplified behavioural space, a social robot must be provided with extrinsic perception in order to interiorize the external world, it must also possess an intrinsic perception system and an imitative learning strategy must be adopted. The process of imitation is innate to humans and plays a crucial role in distinguishing between actions arising from within or actions induced by others, as well as the expression of emotions. Imitation paves the way to the comprehension of the intentions and emotional expressions of others establishing a reciprocal non verbal communication process in which the roles of the imitator and of the model are continuously exchanged. Moreover, in the early years of the life, imitation plays a fundamental role for the emergence of proprioception, of the perception of the external world and of the ability to act our own actions. This is especially evident considering the imitation of emotional expression.

In designing a biomimetic android such as FACE the relationship between mind and body must also be considered. As mentioned, in the previous section, FACE must have an embodied mind, and an interactive relationship with its environment to enable imitative learning and proprioception.

\subsection{The intrinsic perception system}

The FACE artificial sensing skin is a 3D latex foam, under which lies a sensing layer. The sensing layer responds to simultaneous deformations in different directions by means of a piezoresistive network which consists of an Conductive Elastomer (CEs) composites rubber screen printed onto a cotton lycra fabric. CE composites show piezoresistive properties when a deformation is applied and can be easily integrated into fabrics or other flexible substrates to be employed as strain sensors. They are elastic and do not modify the mechanical behaviour of the fabric. CEs consist in a mixture containing graphite and silicon rubber. In the production process of sensing fabrics, a solution of $\mathrm{CE}$ and trichloroethylene is smeared on a lycra substrate previously covered by an adhesive mask. The mask is 
designed according to the desired topology of the sensor network and cut by a laser milling machine. After the deposition, cross-linking process of the mixture is obtained at high temperature. Furthermore, by using this technology, both sensors and interconnection wires can be smeared by using the same material in a single printing and manufacturing process. From technical viewpoint, a piezoresistive sensing fabric is a system whose local resistivity is a function of the local strain. In a discrete way, it can be thought of as a two dimensional resistive network where single resistors have a non-linear characteristic that depends on the local strain. The integral impedance pattern is a function of the overall shape of the sensorized fabric and allows mapping between the electrical space and the shape space. For the characterisation of the sensors in terms of their quasi-static and dynamic electromechanical transduction properties sensors were serially connected. In this case, a current is superimposed in the circuit and high impedance differential voltages are acquired from each sensor. Two multiplexers allow a sensor to be selected and the relative signal is acquired by a differential amplifier. A microprocessor drives the whole system, performs the analogous/digital conversion and exchanges data via an usb interface. The device is provided with an automatic calibration subsystem which allows gain and offset to be tailored to each sensor.

\subsection{The extrinsic perception system}

FACE-T can enable real-time acquisition of both physiological and behavioural information by means of an unobtrusive sensorized wearable interface from the interlocutor. FACE itself is able to analyse the emotional reactions of individuals through optical analyses of facial expressions, to track a human face over time and to automatically store all data (Pioggia et al., 2004). The neuro-computational pre-processing strategy adopted to perceive a subject's face is based on Kohonen Self Organizing Maps (KSOMs). Each KSOM represents a portion of the subject's face (left eye, right eye, nose and mouth) and data of a zone is input to only one map; in this way, each KSOM is trained with the purpose of clustering data coming from the respective zones. The outputs of the KSOMs are used to form the input pattern for the multimodal sensory fusion system.

A wearable interface (life-shirt) for the acquisition of physiological signals from human subjects has been developed at our research labs and integrated into the FACE-T system. The life-shirt approach consists in the integration of smart sensors in a handy garment, together with the integrated electronic devices and the on-body pre-processing of the acquired signals. From a general point of view, the main innovation of this technology lies in the combination of wearable technologies (sensorized garments, electronic sensors, tailored algorithms, on-body computing) together with the user feedback.

The life-shirt within FACE-T collects heterogeneous physiological signals from the human interlocutor of FACE. Acquired signals are transmitted to a server workstation which performs the processing tasks. The life-shirt is based upon three key points: a network of smart sensors, i.e. electrodes and connections embedded on fabric; a wearable acquisition and processing framework equipped with wireless communication systems; a model for data classification, correlation and prediction. Electrodes and connections are woven in the garment, using natural or synthetic fibers containing conductive yarns. A suitable positioning of the electrodes allows physiological signals such as the ECG and the skin electric conductance to be acquired. CE composites rubber screen printed onto fabric have been used to transduce the respiration rhythm. The output of the life-shirt is pre-processed as input vector patterns for the multimodal sensory fusion system, described in the following section. 


\subsection{Multimodal sensory fusion}

A framework for the management and synchronization of data and processes arising from the global FACE-T system has been developed within FACE-T. The framework core and the application processes are interfaced to the sensor array through a framework I/O interface. The framework I/O interface has been developed in order to act as a buffer for the flow of information from the sensors to the application process. Signals coming from different sensor arrays are gathered in parallel and are encoded following a standard protocol. The encoded information is received by a dedicated filter for each sensor, which then sorts them to framework I/O interface. Figure 2 shows the flow of information to and from the framework core.

Communication channels are established as connections between application processes. The domain of data flowing through connections and the flow chart of the application processes can be properly designed according to a specific application. Processes and connections are managed at run time and they can be manipulated under request. The presence of dynamic structures implies a configurable resource management, so the framework offers an optimised interface for enumeration and direct access requests. A spatial definition of the entities involved in the framework can moreover be supplied, making this information available to the control system for subsequent processing. To guarantee the execution of real-time applications an inner synchronization signal is provided from the framework core to the processes and to the framework I/O interface, enabling to gain time-space correlation.

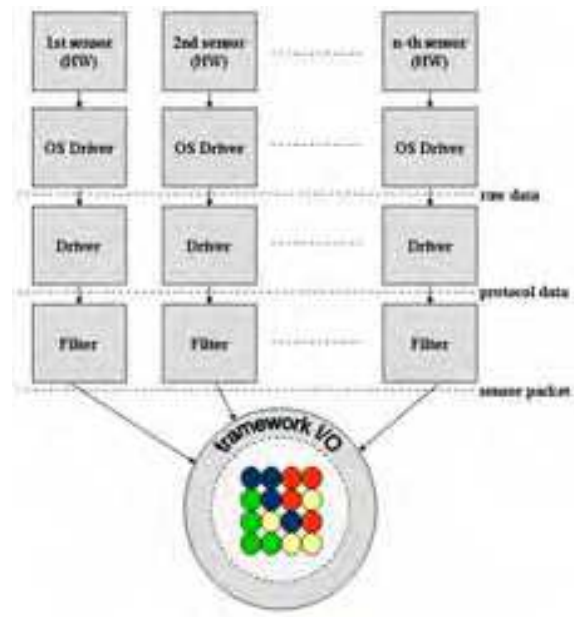

Fig. 2. Framework for the management and synchronization of data and processes.

The framework architecture has been designed as a hierarchical structure whose root is a manager module. It is realised as a high-level container of generic modules representing the environment in which process modules and $\mathrm{I} / \mathrm{O}$ filtering interfaces are placed. Communication channels are realised as connections in specific topologies. All modules are realized as running processes while their control and synchronization is managed by the framework. A real-time approach for data analysis takes advantage of the framework capability to manage interconnected modules through efficient communication channels. In this way the application is able to control all the modules of the processing chain, including analysis protocol management and sensor array interfaces. Connections are delegated to 
dispatch synchronization information and user-defined data. The filtering interface modules are able to drive the transducer hardware and to dispatch information to process modules. All base modules manage dynamic structures and they are designed to maintain data consistency while the environment state may change. This behaviour permits the execution of dynamic and real-time parallel distributed processing while synchronization and data flow are managed by the environment.

This multimodal sensory fusion system manages data and signals from all elements of the FACE-T network allowing dynamic integration of different codes.

\subsection{A neural network for the classification of the behavior of the interlocutor}

In order for the FACE-T system to codify the behavior of the interlocutor, and so interactively adapt FACE to the patient's reactions, a powerful neural network has been implemented.

The complexity of a biological neuron may be reduced by using several mathematical models. Each of these reproduces some of the functionalities of real neurons, such as the excitability in response to a specific input signal. E. Izhikevich (Izhikevich 2003) recently developed a simple model for an artificial neuron which is able to reproduce almost all the functionalities of biological neurons. The model takes 13 FLOPs to simulate one millisecond of neuron activity and it is based on a top-down approach, using two differential equations with four parameters. The computation efficiency and the introduction of axonal delays show the possibility of creating a neural network able to perform real-time classification and prediction tasks (Izhikevich 2005). In this work the Spike-Timing-Dependant Plasticity (STDP) rule (Izhikevich et al. 2004), which permits the implementation of a real time learning rule based on signals which continuously flow from the framework $\mathrm{I} / \mathrm{O}$, has been adopted according to the Theory of Neuronal Group Selection (TNGS) of G. Edelman on selection as the basis for the learning process (Edelman 1993).

The TNGS suggests a novel way for understanding and simulating neural networks. The time variable is taken into account in the learning task, so that neural groups may raise from a selection process. The correspondence between synaptic weights and axonal delays exists as a result of the neuron behavior. One neuron can belong to many groups, and the number of groups is usually higher than the number of the neurons in the map. This guarantees a memory capability which is higher than the capability reached by classical artificial neural networks. The classical approach in artificial neural networks simulation takes into account the modulation of the action potential rhythm as the only parameter for the information flowing to and from each neuron. Such a strategy seems to be in contrast with novel experimental results, since neurons are able to generate action potentials which are based on the input spike timings, with a precision of one millisecond. The spike-timing synchrony is a natural effect that permits a neuron to be activated in correspondence with synchronous input spikes, while the neuronal activation of the post-synaptic neuron is negligible if pre-synaptic spikes arrive asynchronously to the target neuron. Axonal delays usually lie in the range [0.1 44] milliseconds, depending on the type and location of the neuron inside the network. Such a property becomes an important feature for the selection of the neural groups. The selection of neural groups is the result of the variation of synaptic connection according to the STDP rule. If a spike coming from an excitatory pre-synaptic neuron causes the firing of the post-synaptic neuron, the synaptic connection is reinforced since it is given the possibility to generate another spike in order to propagate the signal. Otherwise the synaptic connection is weakened. The values of the STDP parameters are chosen in order to permit a weakening that 
is greater than the reinforcement. Such a strategy permits the progressive removal of unnecessary connections and the persistence of the connections between correlated neurons. The network design is inspired by the anatomical structure found in the mammalian cortex. With respect to the total number $(\mathrm{N})$ of neurons, a percentage equal to $80 \%$ consists of excitatory neurons, while the remaining $20 \%$ are inhibitory neurons. Cortical pyramidal neurons showing a regular spiking behaviour have been adopted for the excitatory subsection, which correspond to appropriate values for the Izhikevich neuron model. Inhibitory neurons have been simulated adopting the model of the cortical interneurons which exhibit fast spiking properties. Each neuron is connected to $\mathrm{M}$ different neurons in order to obtain a connection probability $(\mathrm{M} / \mathrm{N})$ equal to 0.1 , but inhibitory neurons are connected only to excitatory neurons. Moreover, the synaptic weights of the connections arising from the inhibitory neurons remain unchanged during the learning process, while those regarding the connections from the excitatory neurons change according to the STDP rule. Axonal delays are fixed in the range between 1 millisecond and 20 milliseconds. The time resolution has been set to 1 millisecond. The training phase has been carried out for more than 8 hours.

As the application starts, all the connections have the same synaptic weight. The network needs many seconds to get stabilised through depression and strengthening of the synaptic weights. During this first phase, the network shows the presence of a high amplitude rhythm, with frequency in the range between $2 \mathrm{~Hz}$ and $4 \mathrm{~Hz}$ (delta waves).

After a few hours of network activity the spiking rhythm becomes uncorrelated and frequency in the range between $30 \mathrm{~Hz}$ and $70 \mathrm{~Hz}$ appear (gamma waves). The appearance of such rhythms is called PING (Pyramidal-Interneuron Network Gamma) and seems to be related to the spikes of the pyramidal cells which excite the inhibitory interneurons. Such interaction allows a mutual inhibition which temporarily switches-off the network activity. As the network becomes stable, the oscillation rhythm is assessed in the frequency range between $2 \mathrm{~Hz}$ and $7 \mathrm{~Hz}$ and the training phase is ended. The presence of a large number of neural groups can be noted, each able to perform a reproducible spike sequence with a precision of one millisecond. The test phase consists in recording neural group activity in response to predefined FAPs. A labeling procedure allows association of a specific FAP to a neural group. Each FAP is able to select one group inside the network, showing that the network is able to perform classification tasks. Such classification is realised by a memory capability which is far greater than the number of entities involved into the network.

In our opinion, the current neural models do not include the role of glia cells and in particular those of the astrocytes. As has been recently demonstrated, the glia modulates neural communication achieving a two-dimensional continuum in which calcium ion waves influence synaptic communication. The glia cells are the centre of spontaneous activity induced by the continuous rhythm of the oscillations of ions at specific frequencies which influence the coordination and control of neural cells. The complex and dense branching which extends from each astrocyte defines a three-dimensional space, thereby defining an anatomical domain of influence. In the future it is our intention to consider the group of the domains of influence as a single continuous domain, as first suggested by Beurle [Beurle 1956], equipping FACE with a controller, or neurocontroller, made up of groups of neuro-entities placed inside a continuous volume of connected astrocyte cells within an epigenetic topology.

\section{Actions: a behavior-based approach}

Behavior-based control draws inspiration from biology, and tries to model how animals deal with their complex environments and thus forms the basis of FACE's control 
architecture. The components of behavior-based systems are called behaviors: these are observable patterns of activity emerging from interactions between the robot and its environment. Such systems are constructed in a bottom-up fashion, starting with a set of simple behaviors which couple sensory inputs to robot actions. Behaviors are added to provide more complex capabilities. New behaviors are introduced into the system until their interaction results in the desired overall capabilities of the robot. Behavior-based systems and reactive systems share some similar properties: both are built incrementally, from the bottom up, and consist of distributed modules. However, behavior-based systems are fundamentally more powerful, because they can store representations, while reactive systems cannot do so. As mentioned above, an essential prerequisite for imitation is a connection between the sensory systems and the motor systems such that percepts can be mapped onto appropriate actions. In a behavior-based system, the sensing and action oriented behaviors use the same mechanisms and operate on a similar time-scale and representation, enabling the perception of relative and absolute movements, and even the intention of these movements.

\subsection{The cognitive architecture}

The cognitive architecture of FACE is shown in Figure 3. The external world is sensed by FACE and the different stimuli are extracted in terms of neural group activities. In the Perception System, these activities are bound by threshold controlled processes that encode the current set of beliefs about the internal and external state of the android and its relation to the world. The result is a set of response-specific thresholds that serve as antecedent conditions for specific behavioral responses.

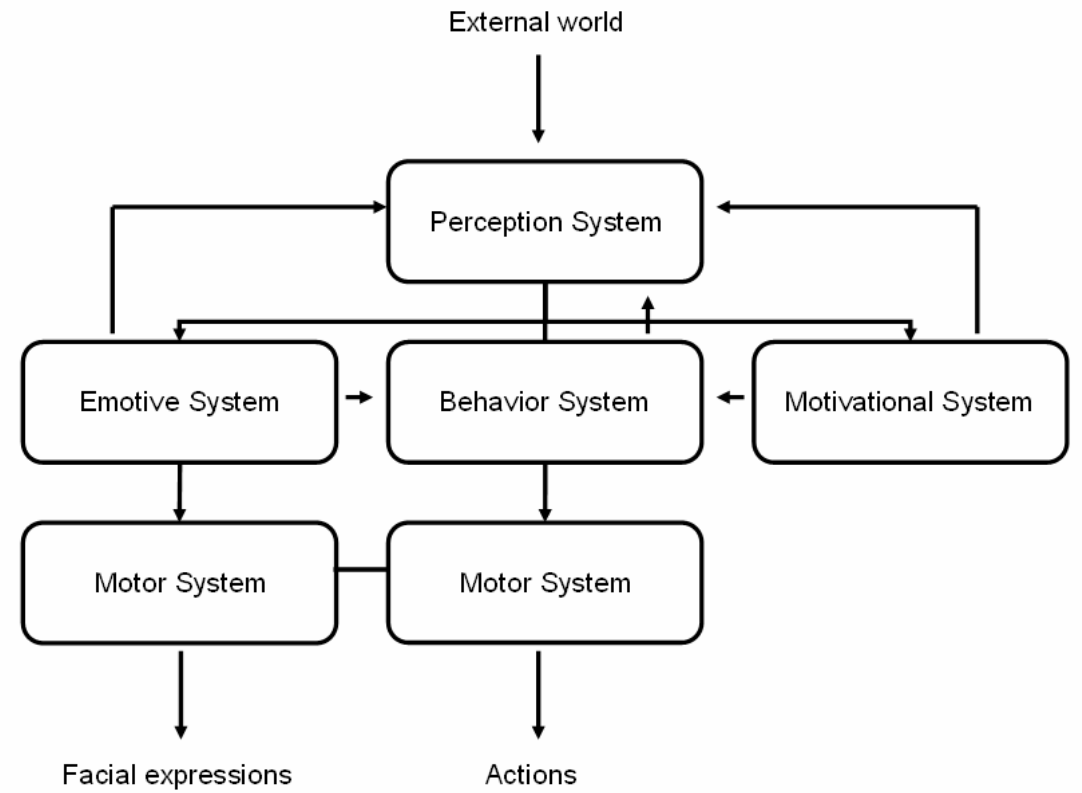

Fig. 3. The cognitive architecture. 
The Emotive System sends a feed-back to the Perception System in order to participate in the evaluation of the stimulus, to the Behavior System in order to participate in the selection of the behavior selection and to the Motor System to activate the facial expression consistent with the emotion. The Motivational System is aimed at influencing the behavior selection. The behaviors participate in the evaluation of the stimulus and they are activated by the Motor System.

\subsection{The perception system}

The perception system analyzes and interprets information from the senses about the outside world. Without a perception system, an individual would be oblivious to any stimuli present in the environment. The perception system is a critical link in making sense of incoming information. It processes the stimuli creating a representation of the surrounding environment on the basis of the following percepts:

- $\quad$ S1 : percept pertinent people's presence

- $\quad$ S2 : percept pertinent physiological data

- $\quad$ S3 : percept pertinent dangerous interaction

- $\quad$ S4 : percept pertinent gaze direction

Activation/deactivation of each percept is represented by means of Boolean values. S1 is activated in case a person is present in the room. S2 is activated when life-shirt is connected. S3 is activated in case of a dangerous interaction with the interlocutor, such as rapid movements of the subject close to FACE. Finally, S4 indicates the occurrence of eye-contact with the subject.

\subsection{The motivational system}

The "needs", or drives, of a machine necessarily influence the behavior selection. They also provide a functional context (i.e. the aim, namely which need the android tries to address) that influences the behavior and perception, as well as the android affective state. Thus, they can also indirectly bias the behavior through the emotion system. Since the drives operate on a slower time scale than the emotions, they contribute to the long-term affective state (or mood of the android) and its expression. The motivational system is based on the following drives corresponding to the first levels of Maslow's Hierarchy of Needs:

- $\quad$ P1 : physiological needs

- P2: safety needs

- P3 : social needs

- P4 : esteem needs

\subsection{The emotive system}

Several theorists argue that a few select emotions are basic or primary: they are endowed by evolution because of their proven ability to facilitate adaptive responses to the vast array of demands and opportunities a creature faces in its daily life (Ekman \& Friesen 1992, Ekman 1992). The emotions of anger, disgust, fear, joy, sorrow, and surprise are often supported as being basic from evolutionary, developmental, and cross-cultural studies (Ekman 1992). Each basic emotion serves a particular function (often biological or social), arising in particular contexts, to prepare and motivate a creature to respond in adaptive ways. They serve as important reinforcers for learning new behavior. In addition, emotions are refined 
and new emotions are acquired throughout emotional development. Emotions seem to be centrally involved in determining the behavioral reaction to environmental (often social) and internal events of major significance for the needs and goals of a creature (Izard 1993). The emotive system of FACE is based on the following emotions:

- $\quad$ E1 : enjoyement

- E2 : sadness

- E3 : anger

- E4 : disgust

- E5 : fear

- E6 : surprise

\subsection{The behaviors}

Every behavior is modelled as a separate goal-directed process. In general, both internal and external factors are used to compute their relevance (whether or not they should be activated). The behavior system allows FACE to respond to a stimulus and to fulfil the aim. Once the stimulus is evaluated by the perception, motivational and emotive systems, the expected behavior, present in the following list, must be activated on the basis of the aim, of the environment and on the security needs:

- B1 : self preservation

- B2 : quite

- B3 : gaze

- B4 : joint attention

- B5 : communication (imitation)

B1 is devoted to the security needs. It can be activated on the basis of an evaluation of the motivational system and/or on the basis of emotive system. B2 indicates a warning. B3 and $\mathrm{B} 4$ activate respectively the eye contact and the joint attention. B5 enables the imitation task.

\subsection{The motor system}

The motor system directly implements the following actions in FACE:

- A1 : attention

- A2 : rest

- A3 : quite expression

- A4 : ocular movements

- A5 : head movements

- A6 : facial expressions

Each action is a collection of motor primitives that directly drives each motor by applying voltage pulses. During the pulse application the selected motor applies an increasing force, while, during the rest phase the motor is floating.

\section{Experimental results}

A block schema of the experimental set-up for the FACE-T system is shown in figure 4 . It consists of a specially equipped room, provided with two remotely orientable video cameras, in which the child, under the supervision of a therapist, can interact with FACE by means of a liquid crystal screen, a keyboard and a mouse. Both FACE and the interactive 
module are connected to a computer. The subject wears the life-shirt for recording physiological data. The database also contains data from the audio visual recording system present in the room. Other therapists or Hidden Observers compile evaluation sheets during sessions, and the data scanned from these will also be added to the database and used for successive analysis.

In order to obtain a preliminary evaluation of the behavior of children affected by ASD when exposed to FACE, we set up an experimental session in which the reactions of 4 subjects ( 3 male and 1 female), between 7 and 20 years old, were monitored and compared. The children with autism had been diagnosed using ADI-R and ADOS-G, two specific diagnostic instruments, as high functioning autism, and are currently under treatment at the Stella Maris Institute (IRCCS) in Pisa, Italy.

\begin{tabular}{|c|c|c|}
\hline Subjects & Age & IQ \\
\hline S1 & $10 \mathrm{y} 6 \mathrm{~m}$ & 105 \\
\hline S2 & $9 \mathrm{y} 6 \mathrm{~m}$ & 87 \\
\hline S3 & $8 \mathrm{y} 11 \mathrm{~m}$ & 85 \\
\hline S4 & $20 \mathrm{y} 6 \mathrm{~m}$ & 52 \\
\hline
\end{tabular}

Table 1. Autism rating scale for the selected subjects.

Experiments were carried out in order to study the interaction with FACE during twenty minute sessions. We studied:

- both spontaneous behavior of the participants and their reactions to therapist presses in correlation with the time course of the physiological and behavioural data

- the focusing of the attention towards FACE's eye movements

- the spontaneous ability of imitation of gesture and expressions of the android

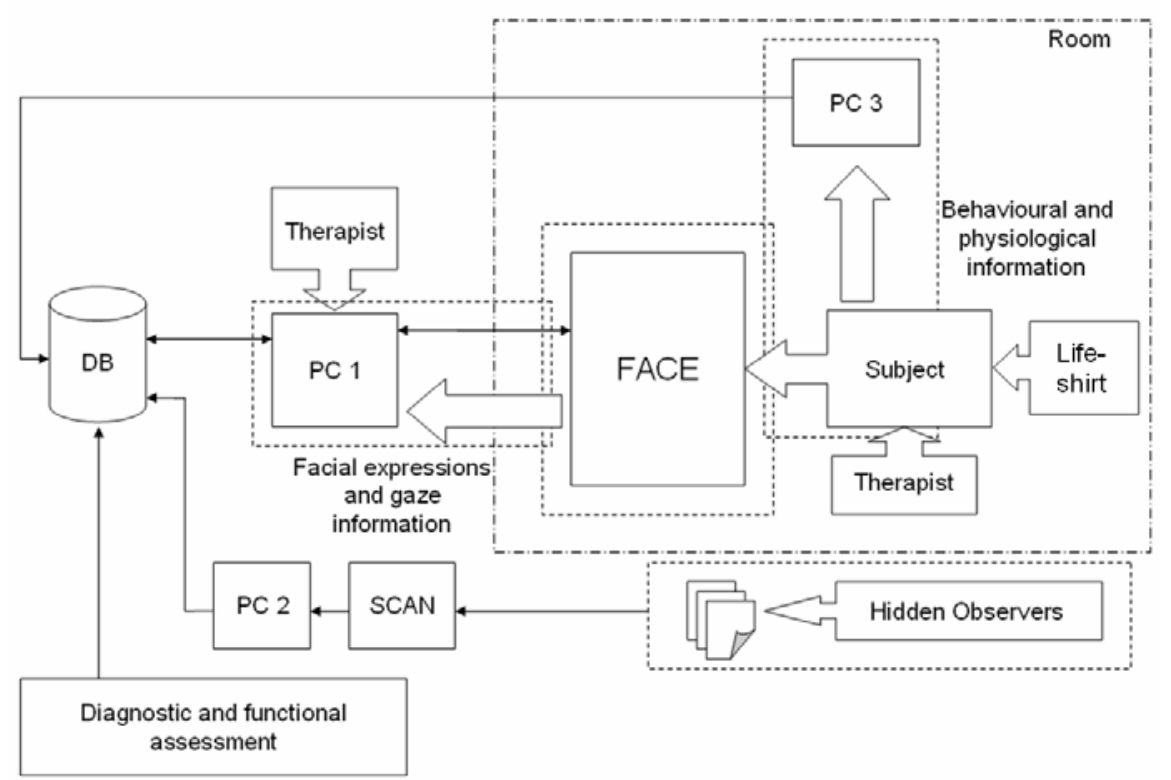

Fig. 4. Experimental set-up for implementing FACE-T. 
The evaluation of the treatment effect was performed using 8 relevant items from the CARS scale.

Figures 5, 6, 7 and 8 supply a graphical comparison between the score obtained in items of CARS scale in previous interactions during psychological treatments and the one obtained with FACE. In particular, we observed that the CARS score decreased or remained the same for all items as regards subjects 2 and 3 after the therapy session. Only subject 4 (the oldest, with lowest IQ and highest ADOS rating) showed an increase of 0.5 points for listening, fear and verbal communication. More importantly, all the subjects demonstrated a decrease in the score of emotional response in the CARS scale of between 1 and 0.5 points, and imitation in 3 out of 4 children, so implying a marked improvement in these areas after interacting with FACE. Even though these are the first set of clinical trials, it is clear that the presence of FACE in a therapeutic environment can lead to improvements in the areas of social communication and imitation. As shown in figure 9, the cardiac frequency of the patient increases after his attention is focused on the robot, and remain fairly high till he is forced to focus on his emotional relationship with FACE.

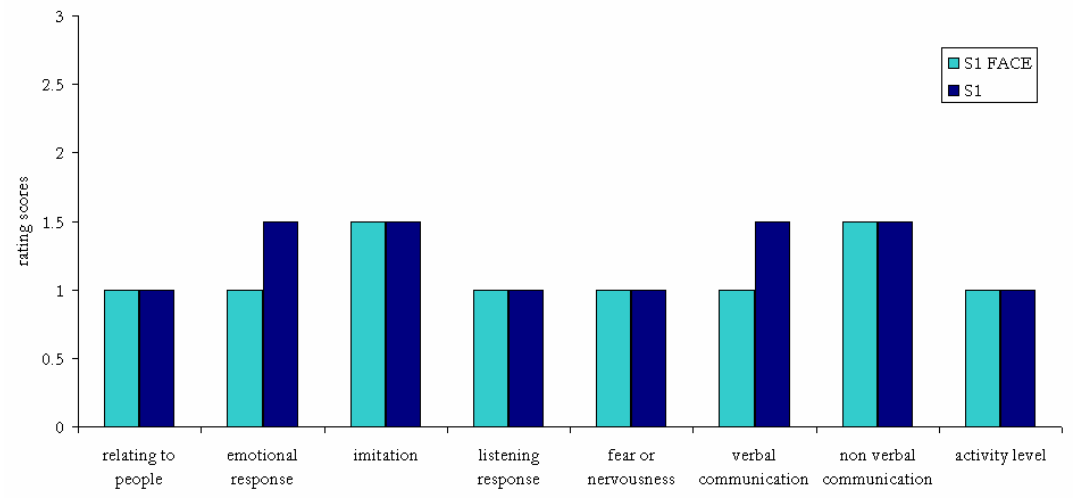

Fig. 5. Subject 1 - S1 FACE: CARS score obtained from the experimental session with FACE. S1: CARS score obtained from the observation of session with psychological tests.

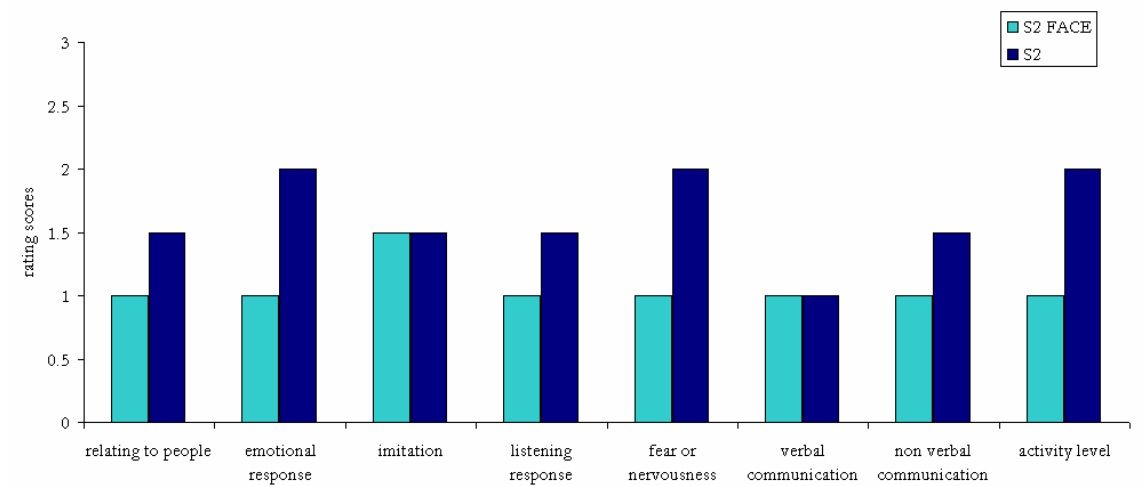

Fig. 6. Subject 2 - S2 FACE: CARS score obtained from the experimental session with FACE. S2: CARS score obtained from the observation of session with psychological tests. 


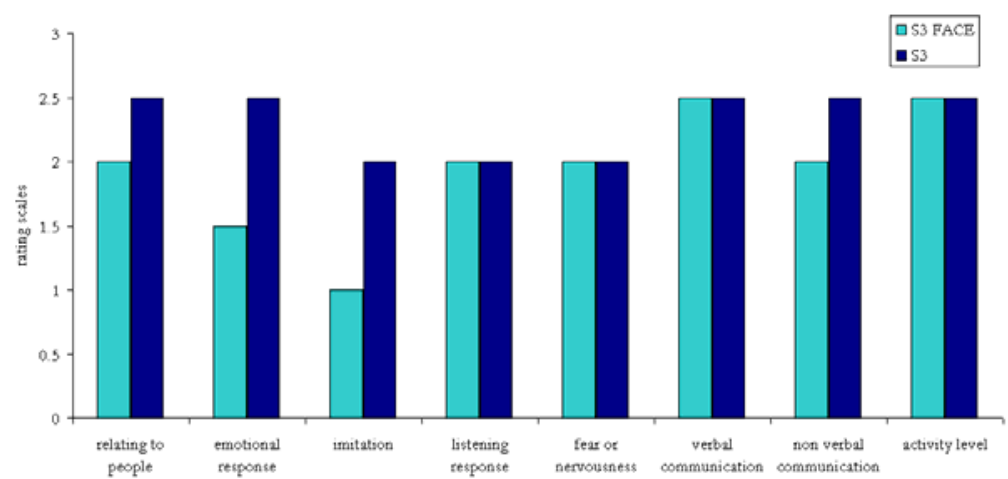

Fig. 7. Subject 3 - S3 FACE: CARS score obtained from the experimental session with FACE. S3: CARS score obtained from the observation of session with psychological tests.

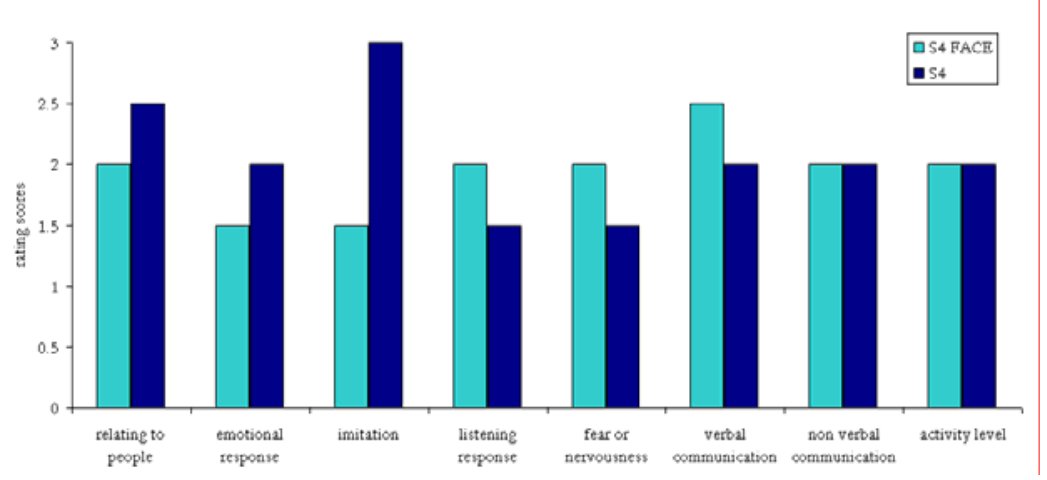

Fig. 8. Subject 4 - S4 FACE: CARS score obtained from the experimental session with FACE. S4: CARS score obtained from the observation of session with psychological tests.

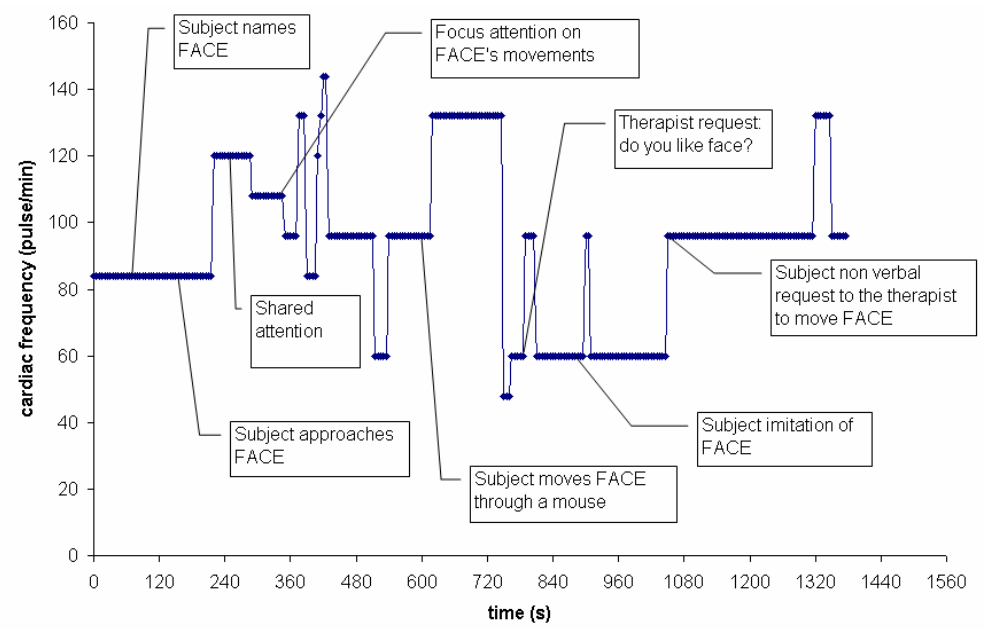

Fig. 9. Typical trace of a subject's heart rate during the treatment. 
Figures 10, 11 and 12 show snapshots of an experimental session. In figure 10 the subject shows is shown to completely focus his attention on FACE. In figure 11 a spontaneous approaching of the subject for eye contact with FACE is shown. While figure 12 shows the non verbal requesting of the subject through a conventional gesture (a wink).

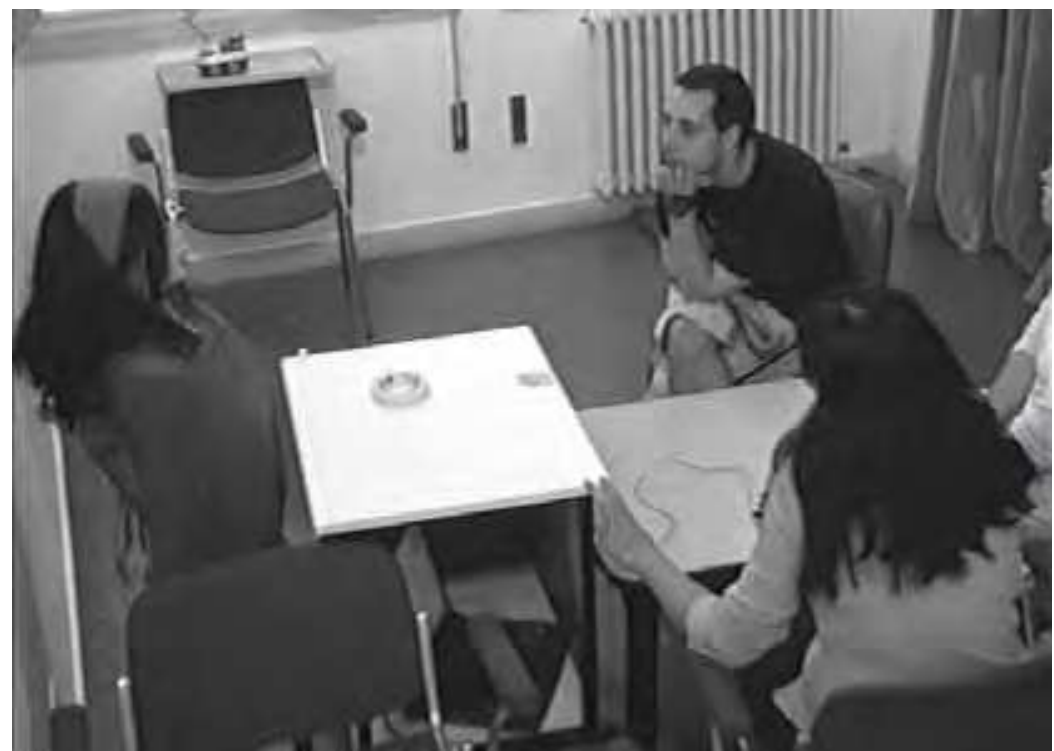

Fig. 10. Experimental session: focus of attention on FACE (S4).

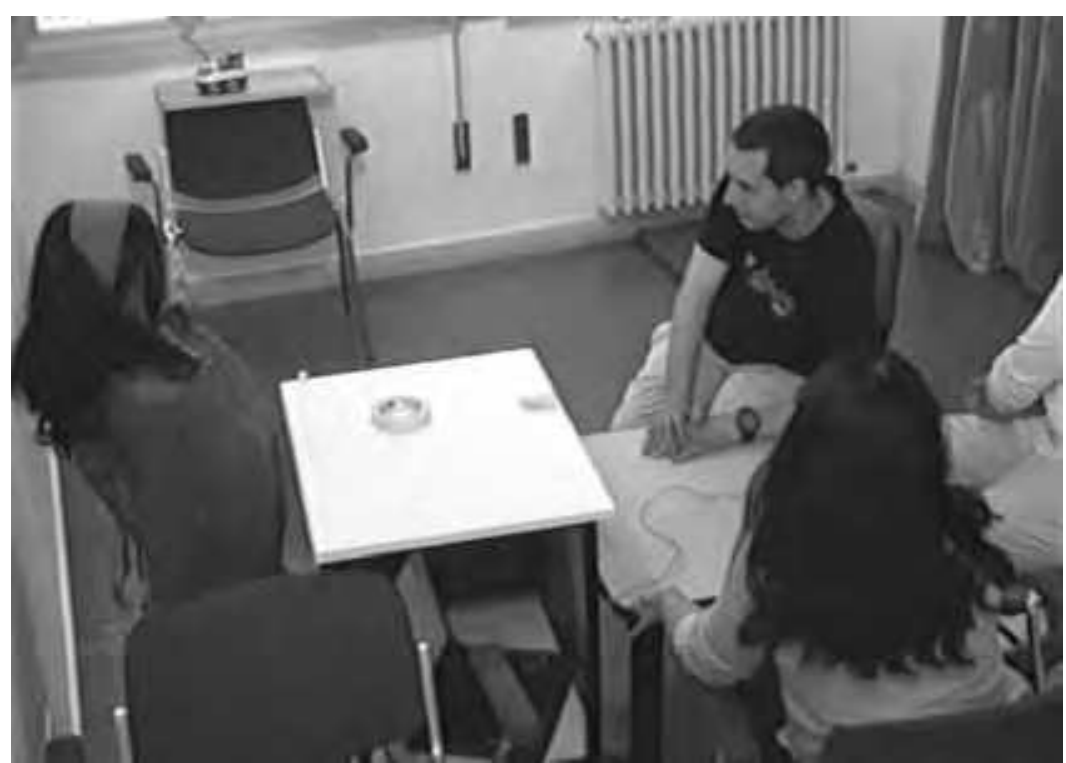

Fig. 11. Experimental session: spontaneous approaching for eye contact with FACE (S4). 


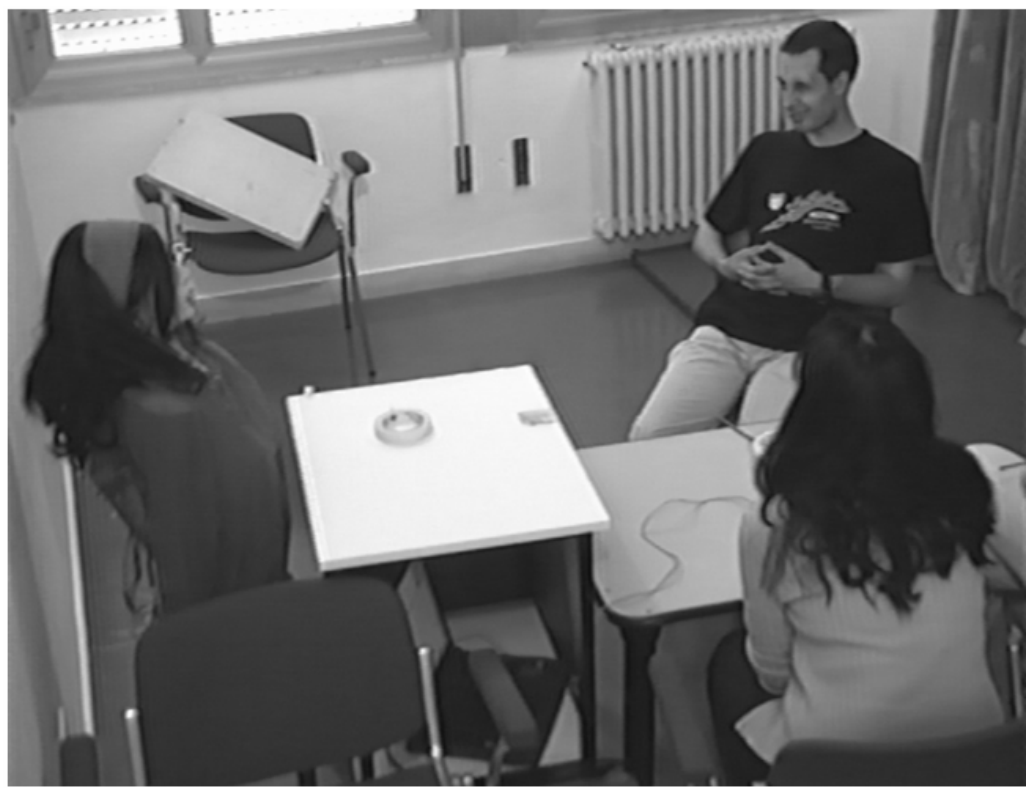

Fig. 12. Experimental session: non verbal requesting through conventional gesture (S4).

The therapeutic trails are still in preliminary phases. Nevertheless, all 4 subjects as well as controls show no fear in the presence of FACE, and all autistic subject showed same improvement in CARS scores, particularly as regards imitation, communication and emotional response.

Future work in this direction will focus on identifying specific criteria for evaluation the subject response, on conducting a larger range of trails, and on repeating treatment monitor signs of progress in patients. These initial results illustrate the validity of the android based FACE-T approach in social and emotive treatments in ASD. We believe that its potency lies in the fact that FACE is based primarily on learning by imitation while deficits in imitation are implicated in ASD.

\section{Conclusion}

The aim of FACE-T is to act as a human-machine interface for non verbal communication. The learning process in FACE is based on imitating predefined stereotypical behaviours which can be represented in terms of FAPs followed by a continuous interaction with its environment. At present FACE is applied to enhance social and emotive abilities in children with autism. The experimental sessions allowed us to collect preliminary data in terms of therapeutic treatment for patients with disorders in the autistic spectrum. However, what is behind FACE? There is the application of the smart soft matter, algorithms and robotics, there is the attempt to understand the complexity of biological behaviour, there are people with autism. During a test, little M., following the movements of FACE's eyes, noticed that FACE looked at him again and a little bit surprised, upon a request of the therapist to give a meaning to this situation, said "I exist too and therefore I'm important". What is behind FACE?. 


\section{Acknowledgments}

Authors would like to thank the Academy of Fine Arts of Carrara, Italy, http://www.accademiacarrara.it, in the persons of Director Baudinelli and Prof. Marchetti for the work performed on the latest prototype of FACE.

\section{References}

American Psychiatric Association (1994). Diagnostic and statistical manual (4th ed.). Washington, DC: Author.

Baron-Cohen, S.; Ring, H.; Bullmore, E.; Wheelwright, A.C. \& Williams, S.C.R. (2000). The amygdala theory of autism. Neuroscience and Behavioral Reviews. 24, 355-364.

Baron-Cohen, S. (1997). Mindblindness: An Essay on Autism and Theory of Mind. Cambridge, MA: MIT Press.

Beurle, R.L. (1956). Properties of a mass of cells capable of regenerating pulses. Phil. Trans. R. Soc. A240, 5597.

Billard, A. (2002). Play, Dreams and Imitation in Robota. In K. Dautenhahn, B. Edmonds and L. Canamero (eds), Socially Intelligent Agents, Kluwer publisher, 165-173.

Breazeal (Ferrell), C. \& Scassellati, B. (2000). Infant-like Social Interactions Between a Robot and a Human Caretaker in Special issue of Adaptive Behavior on Simulation Models of Social Agents, guest editor Kerstin Dautenhahn.

Dapretto, M.; Davies, M.S.; Pfeifer, J.H.; Scott, A.A.; Sigman, M.; Bookheimer, S.Y. \& Iacoboni, M. (2005). Understanding emotions in others: mirror neuron dysfunction in children with autism spectrum disorders, Nature Neuroscience 9, 28 - 30.

Dautenhahn, K. (2002a). Design Spaces and Niche Spaces of Believable Social Robots. Proceedings IEEE Ro-man 2002 International Workshop on Robot and Human Interactive Communication, September 25-27, Berlin, Germany, pp. 192-197, IEEE Press.

Dautenhahn, K.; Werry, I.; Rae J. \& al., (2002b). Robotic playmates: analysing interactive competencies of children with autism playing with a mobile robot. In Dautenhahn K, Bond A, Canamero L et al, eds. Socially intelligent agents creating relationships with computers and robots. Kluwer Academic Publishers. 117-124.

Davies, S.; Bishop, D.; Manstead, A.S.R. \& Tantam, D. (1994). Face Perception in children with autism and Aspergers Syndrome. Journal of Child Psychology and Psychiatry, 35, 1033-1057.

Dawson, G.; Meltzoff, A.; Osterling, J. \& Rinaldi, J. (1998). Neuropsychological correlates of early symptoms of autism. Child development, 69, 1277-1285.

Decety, J.; Perani, D.; Jeannerod, M.; Bettinardi, V.; Tadary, B.; Woods, R.; Mazziotta, J.C. \& Fazio, F. (1994). Mapping motor representations with positron emission tomography. Nature, 371, 600-602.

Decety, J. (1996). Do imagined and executed actions share the same neural substrate? Cognitive Brain Research, 3, 87-93.

Edelman, G.M. (1993). Bright Air, Brilliant Fire: On the Matter of the Mind. Basic Books; Reprint edition.

Ekman, P. (1992). Are there basic emotions? Psychological Review 99 (3), 550-553

Ekman, P. \& Friesen, W. (1982). Measuring facial movement with the facial action coding system, In: Ekman, P. (Ed.), Emotion in the Human Face. Cambridge University Press, Cambridge, UK, pp. 178-211. 
Fadiga, L.; Fogassi, L.; Pavesi, G. \& Rizzolatti, G. (1995). Motor facilitation during action observation: A magnetic stimulation study. J. Neurophysiol., 73, 2608-2611.

Gallese, V. \& Goldman, A. (1998). Mirror neurons and the simulation theory of mindreading, Trends in Cognitive Sciences Vol . 2, No. 12.

Klein, A.; Warren Jones, BA.; Schultz, R.T.; Volkmar, F.R. \& Cohen, D. (2002). Visual Fixation Patterns During Viewing of Naturalistic Social Situations as Predictors of Social Competence in Individuals with Autism. Arch Gen Psychiatry. 59: 809-816.

Kozima, H.; Nakagawa, C. \& Yano, H. (2004). Can a robot empathize with people?, International Journal Artificial Life and Robotics Vol.8, pp.83-88.

Kozima, H. (2002). Infanoid: A babybot that explores the social environment, K. Dautenhahn, A. H. Bond, L. Canamero, B. Edmonds (eds.), Socially Intelligent Agents: Creating Relationships with Computers and Robots, Amsterdam: Kluwer Academic Publishers, pp.157-164.

Izard, C. (1993). Four systems for emotion activation: cognitive and non cognitive processes, Psychological Review 100, 68-90

Izard, C. (1977). Human Emotions, Plenum Press, New York, NY.

Izard, C. (1994). Cognition is one of four types of emotion activating systems, In: Ekman, P., Davidson, R. (Eds.), The Nature of Emotion. Oxford University Press, New York, NY, pp. 203-208.

Izhikevich, E.M. (2003). Simple model of spiking neurons. IEEE transaction on neural networks, vol. $15, \mathrm{n}^{\circ} 6$.

Izhikevich, E.M. (2005), Polycronization: computation with spikes. Neural Computation.

Izhikevich, E.M.; Gally J.A. \& Edelman G.M. (2004). Spike-timing dynamics of neural groups. Cerebral Cortex, 14:933-944

Lord, C. ; Rutter, M. \& Le Couter, A. (1994). Autism Diagnostic Interview-revised: A revised version of a diagnostic interview for caregivers of individuals with possible pervasive developmental disorders. Journal of Autism and Developmental Disorders 24, 659-686.

Lord, C.; Risi, S. ; Lambrecht, L. ; Cook, E.H. ; Leventhal, B.L. \& al. (2000). The Autism Diagnostic Observation Schedule-Generic: A standard measure of social and communication deficits associated with the spectrum of autism. Journal of Autism and Developmental Disorders, 30, 205-223.

Maestro, S.; Muratori, F.; Cavallaro, M.C.; Pei, F.; Stern, D.; Golse, B. \& Palacio-Espasa, F. (2002). Attentional skills during the first 6 months of age in autism spectrum disorder. J Am Acad Child Adolesc Psychiatry, 41(10):1239-45.

Mataric, M. (2002). Situated Robotics, Encyclopedia of Cognitive Science, Nature Publishers Group, Macmillian Reference Ltd.,

Michaud, F.; Duquette, A. \& Nadeau, I. (2003). Characteristics of mobile robotic toys for children with Pervasive Developmental Disorders. Proceedings IEEE Conference on Systems, Man, and Cybernetics.

Michaud, F. \& Théberge-Turmel, C. (2002). Mobile robotic toys and autism. In Dautenhahn $\mathrm{K}$, Bond A, Canamero L et al, eds. Socially intelligent agents creating relationships with computers and robots. Kluwer Academic Publishers, 125-132.

Meltzoff, A.N. \& Moore, M.K. (1995). Infant's understanding of people and things: From body imitation to folk psychology. In J. L. Bermúdez, A. Marcel, \& N. Eilan (Eds.), The Body and the Self (pp. 43-69). Cambridge, MA: MIT Press.

Meltzoff, A.N. \& Moore, K.M. (1997). Explaining Facial Imitation: A theoretical Model, Early development and Parenting, Vol 6, 179-192. 
Nadel, J. ; Revel, A. ; Andry, P. \& Gaussier P. (2004a). Towards communications: first imitation in infants, children with autism and robots. Interaction Studies. 1, 45-75.

Nadel, J., (2004b). Early imitation and the emergence of a sense of agency. Proceedings of the Fourth International Workshop on Epigenetic Robotics, Genova, Italy.

O'Reilly, R.C. \& Munakata Y. (2000). Computational Exploration in Cognitive Neuroscience: Understanding the Mind by Simulating the Brain. Bradford Book, MIT Press, Cambridge.

Pioggia, G.; Ahluwalia, A.; Carpi, F.; Marchetti, A.; Ferro, M.; Rocchia, W. \& De Rossi, D. (2004). FACE: Facial Automaton for Conveying Emotions. Applied Bionics and Biomechanics. 1(2), 91-100.

Pioggia, G.; Igliozzi, R.; Ferro, M.; Ahluwalia, A.; Muratori, F. \& De Rossi, D. (2005). An Android for Enhancing Social Skills and Emotion Recognition in Autistic patients. IEEE Transaction on Neural System and Rehabilitation Engineering. 3(4), 507-515.

Rizzolatti, G.; Fadiga, L.; Fogassi, L. \& Gallese, V. (1999). Resonance behaviors and mirror neurons. Archives of Italian Biology. 137, 85-100.

Rizzolatti, G. \& Arbib, M.A. (1998). Language within our grasp. Trends Neurosci., 21, 188-94.

Schopler, E.; Reichler, R.J. \& Renner, B.R. (1988). The Childhood Autism Rating Scale. Los Angeles: Western Psychological Services.

Smith I. M., Bryson S.E. Imitation and action in autism: A critical review. Psychology Bulletin, 1994, 116, 259-273.

Spelke, E.S.; Phillips, A. \& Woodward, A.L. (1995). Infants Knowledge of objects motion and human action. In: Sperber D., Premack D., Premack AJ., eds causal Cognition: A Multidisciplinary Debate. Oxford, England: Oxford University Press; 44-78.

Schultz, R.T.; Gauthier, I.; Klin, A.; Fulbrigth, R.; Anderson, A.; Volkmar, F.R.; Skudlarski, P.; Lacadie, C.; Cohen, DJ. \& Gore, J.C. (2000). Abnormal ventral temporal cortical activity among individuals with autism and asperger syndrome during face discrimination. Arch Gen Psychiatry. 57: 331-340.

Tager-Flusberg, H. \& Cohen, D.J. (1994). Understanding other minds: Perspectives from autism. New York: Oxford University Press.

Weir, S. \& Emanuel, R. (1976). Using Logo to Catalyse Communication in an Autistic Child. D.A.I. Research Report, University of Edinburgh.

World Health Organization (1993). International classification of diseases. (10th ed., Draft version). Geneva. 


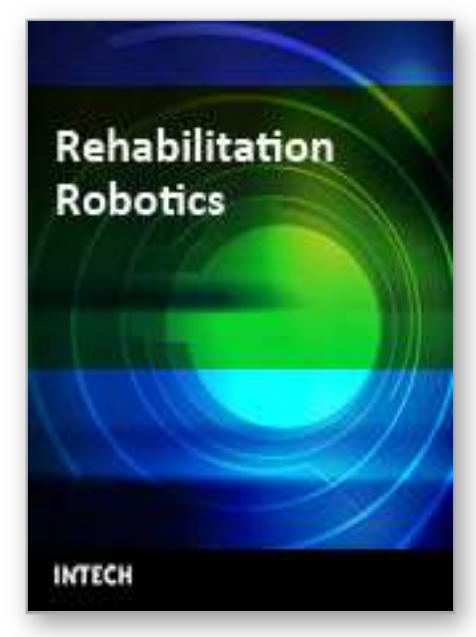

\author{
Rehabilitation Robotics \\ Edited by Sashi S Kommu
}

ISBN 978-3-902613-04-2

Hard cover, 648 pages

Publisher I-Tech Education and Publishing

Published online 01, August, 2007

Published in print edition August, 2007

The coupling of several areas of the medical field with recent advances in robotic systems has seen a paradigm shift in our approach to selected sectors of medical care, especially over the last decade.

Rehabilitation medicine is one such area. The development of advanced robotic systems has ushered with it an exponential number of trials and experiments aimed at optimising restoration of quality of life to those who are physically debilitated. Despite these developments, there remains a paucity in the presentation of these advances in the form of a comprehensive tool. This book was written to present the most recent advances in rehabilitation robotics known to date from the perspective of some of the leading experts in the field and presents an interesting array of developments put into 33 comprehensive chapters. The chapters are presented in a way that the reader will get a seamless impression of the current concepts of optimal modes of both experimental and ap- plicable roles of robotic devices.

\title{
How to reference
}

In order to correctly reference this scholarly work, feel free to copy and paste the following:

Giovanni Pioggia, Maria Luisa Sica, Marcello Ferro, Silvia Casalini, Roberta Igliozzi, Filippo Muratori, Arti Ahluwalia and Danilo De Rossi (2007). Facial Automaton for Conveying Emotions as a Social Rehabilitation Tool for People with Autism, Rehabilitation Robotics, Sashi S Kommu (Ed.), ISBN: 978-3-902613-04-2, InTech, Available from:

http://www.intechopen.com/books/rehabilitation_robotics/facial_automaton_for_conveying_emotions_as_a_so cial_rehabilitation_tool_for_people_with_autism

\section{INTECH}

open science | open minds

\author{
InTech Europe \\ University Campus STeP Ri \\ Slavka Krautzeka 83/A \\ 51000 Rijeka, Croatia \\ Phone: +385 (51) 770447 \\ Fax: +385 (51) 686166 \\ www.intechopen.com
}

\author{
InTech China \\ Unit 405, Office Block, Hotel Equatorial Shanghai \\ No.65, Yan An Road (West), Shanghai, 200040, China \\ 中国上海市延安西路65号上海国际贵都大饭店办公楼 405 单元 \\ Phone: +86-21-62489820 \\ Fax: +86-21-62489821
}


(C) 2007 The Author(s). Licensee IntechOpen. This chapter is distributed under the terms of the Creative Commons Attribution-NonCommercial-ShareAlike-3.0 License, which permits use, distribution and reproduction for non-commercial purposes, provided the original is properly cited and derivative works building on this content are distributed under the same license. 